\title{
Dynamique du phytoplancton en relation avec certains paramètres physico-chimiques dans la retenue El Kansera (Maroc)
}

\author{
D. Fqih Berrada 1 \\ R. Berrada 1 \\ A. Benzekri²
}

Mots-clés : phytoplancton, dynamique, cyanobactéries, diatomées, retenue de barrage.

L'étude de la dynamique saisonnière du phytoplancton dans la retenue El Kansera a été réalisée dans le but de comprendre au mieux le phénomène d'eutrophisation de cet écosystème lacustre. La biomasse (exprimée en poids frais) a été suivie pendant un cycle et demi, d'avril 1995 à août 1996. Cette période étant marquée par la transition d'une année de sécheresse avec une année pluvieuse, la composition et la dynamique saisonnière des peuplements algaux est analysée en fonction des variables environnementales. En 1995, année de sécheresse, le peuplement algal est dominé par les cyanobactéries, en particulier Microcystis aeruginosa qui représente plus de $99 \%$ de la biomasse algale totale. En 1996, année pluvieuse, le peuplement algal est plus diversifié et constitué d'une diatomée centrique (Cyclotella ocellata) et de Chlorococcales (Crucigenia apiculata, Scenedesmus ecornis, Oocystis lacustris...). La saison d'été est marquée en revanche par la recolonisation du milieu par M.aeruginosa. Les variables environnementales (climat, physico-chimie de l'eau et apports exogènes) semblent régir de près la succession phytoplanctonique dans la retenue El Kansera.

Dynamics of phytoplankton and relationship with physical and chemical parameters in El Kansera lake-reservoir (Morroco)

Keywords : phytoplankton, dynamics, cyanobacteria, diatoms, lake-reservoir.

A study of the seasonal dynamics of the phytoplankton in the El Kansera lake-reservoir has been carried out in order to understand the eutrophication process of this lake. Biomass (wet weight) was monitored from April 1995 to August 1996. This period is characterised by the transition from a dry year to a rainy year. So, composition and dynamics of phytoplankton are analysed, and related to environmental factors. In 1995 cyanobacteria dominated the algal communities ; Microcystis aeruginosa represented more than $99 \%$ of the total biomass. In 1996, phytoplankton was more diversified and constituted of a centric diatom (Cyclotella ocellata) and chlorococcals (Crucigenia apiculata, Scenedesmus ecornis, Oocystis lacustris...). In summer, M.aeruginosa appeared again and dominated the algal communities. Environmental factors (climate, water composition and exogenic inputs) seem to control appreciably the phytoplankton succession in El Kansera lake-reservoir.

\section{Introduction}

Les études consacrées aux peuplements phytoplanctoniques des systèmes limniques en climat chaud sont

1. Université Hassan II, Faculté des Sciences Aïn Chock I de Casablanca, Département Biologie, B.P. 5366, Maârif, Maroc. 2. Laboratoire Public d'Essais et d'Etudes de Casablanca, Centre d'Etudes et de Recherches sur la pollution de l'Eau et de l'Air. Centre d'activité Oukacha, Bd Moulay Slimane, AÏn Sebâa, Maroc. encore peu nombreuses, comparées à celles des régions tempérées (Capblancq 1972, Lair \& Sargos 1981, Dauta 1982, Devaux et al. 1983, Reynolds 1984, - Lampert et al. 1986, Jrad 1989, Balvay et al. 1990, Gala \& Giesy 1991, Le Cohu et al. 1991, Köhler 1992, Varis 1993, Quiblier-Lloberas et al. 1996, Enrisken \& Moestryp 1997...).

Au Maroc, ce n'est que ces dix dernières années que plusieurs recherches ont été entreprises sur des sites variés tels que cours d'eau et bassins (Mouhri 1987, Essini 1987, Maiffi 1988). Parallèlement, les travaux consacrés aux divers aspects de l'écologie du phyto- 
plancton dans les retenues de barrage se sont diversifiés tout en restant limités (Abouzaid \& Foutlane 1986, Bouterfas 1990, Loudiki 1990, Lahlou 1991, Hasnaoui 1992, Cherifi 1992, Malki 1994, Derraz 1995).

L'objectif de cette étude est de caractériser le peuplement algal et d'analyser ses variations spatio-temporelles dans la retenue de barrage El Kansera, située au Nord-Ouest du Maroc. Cet ouvrage assure l'approvisionnement en eau potable des 2 villes Khemisset et Tiflet, une production électrique annuelle de 15000 $\mathrm{KWh}$, l'irrigation de 25.000 ha et la protection contre les crues de l'oued Beht, dernier affluent important de l'oued Sebou. La période d'étude (avril 1995-août 1996) a été marquée par de fortes variations climatiques (passage d'une année de sécheresse à une année pluvieuse), qui se sont répercutées sur la dynamique des peuplements phytoplanctoniques de la retenue. Nos résultats essayent de démontrer l'impact des paramètres environnementaux - climat, physico-chimie de l'eau et apports allochtones de l'oued Beht - sur la composition, la variabilité et la distribution spatiotemporelle du phytoplancton.

\section{Méthodes et principales caractéristiques du site d'étude}

L'échantillonnage du phytoplancton a été effectué selon une périodicité mensuelle, à différentes profondeurs de la station barrage (Fig. 1). En raison des fortes variations climatiques durant la période d'étude qui se sont répercutées sur le niveau du plan d'eau de la retenue (Fig. 2), les niveaux prospectés différent d'une année à l'autre. En 1995, les profondeurs étudiées sont $-0,5,2,6$ et $10 \mathrm{~m}$, alors qu'en 1996,2 nouvelles profondeurs s'ajoutent $(-15,-20 \mathrm{~m})$. Les prélèvements d'eau ont été réalisés à l'aide d'une bouteille à colonne verticale en P.V.C, de type Van Dorn (Lamotte \& Bourlière 1971). La fixation des algues a été réalisée au lugol. Le comptage a été effectué au microscope inversé (Uthermöhl 1958). La biomasse est exprimée en poids frais, déterminé après estimation du volume des cellules (Lohmann 1908). Dans le cas de Microcystis aeruginosa Kütz, le biovolume a été effectué à partir des colonies (Fig.3).

Les analyses physico-chimiques de l'eau ont été réalisées selon les normes en vigueur, au laboratoire public d'essais et d'études de Casablanca, agrée par le comité scientifique français d'accréditation (COFRAC). La transparence de l'eau et la zone euphotique ont été évaluées à l'aide du disque de Secchi (Reynolds 1984). Les nitrates ont été dosés par colorimétrie après réduction sur colonne de cadmium, le phosphore total après minéralisation en milieu acide, les orthophosphates et la silice en présence de molybdate d'ammonium, selon les normes Afnor (1994).

Le lac-réservoir El Kansera est de type monomictique chaud, avec une stratification thermique et chimique (oxygène), relativement stables du mois d'avril à mi-septembre (Fig.4). Les principales caractéristiques de la retenue sont données dans le tableau $1 . \mathrm{La}$ figure 2 décrit l'évolution temporelle de la transparence de l'eau et de la zone euphotique en fonction du niveau du plan d'eau de la retenue. Les figures 5 et 6 décrivent les cycles annuels de l'azote nitrique (N-NO3), du phosphore total (PT), des orthophosphates (P-PO4), de la silice $(\mathrm{SiO} 2)$ et du rapport N-NO3/P-PO4.

\section{Résultats}

\subsection{Variations saisonnières de la biomasse algale}

L'évolution saisonnière de la biomasse algale (poids frais) dans la zone euphotique montre d'importantes variations, tant saisonnières qu'annuelles (Fig.7). L'année 1995 est particulièrement marquée par deux pics :

- le premier au mois de juin (73 $\mathrm{mg}^{-\mathrm{l}^{-1}}$ ), est imputable à la cyanobactérie Microcystis aeruginosa Kütz, qui représente $99 \%$ de la biomasse totale. D'autre cyanobactéries se développent en cette période de façon secondaire, telles que Phormidium sp. Kütz., Aphanizomenon gracile Lemme, Lyngbya lutea Agardh, Oscillatoria tenuis Agardh ou Merismopedia punctata Meyen ;

- le second pic au mois d'octobre ( $\left.34 \mathrm{mg} . \mathrm{l}^{-1}\right)$, est lié à un nouveau bloom de Microcystis.

En 1996, deux autres pics de biomasse sont enregistrés :

- le premier au mois d'avril $\left(9,46 \mathrm{mg} . \mathrm{l}^{-1}\right)$ est représenté essentiellement par la diatomée Cyclotella ocellata Pant. $(80 \%)$ et la chlorococcale Crucigenia apiculata Schmidle (20\%);

— le second au mois de juillet $\left(72,7 \mathrm{mg} . \mathrm{l}^{-1}\right)$ est dû à un nouveau bloom de Microcystis aeruginosa ( $83 \%$ ). Au même moment, d'autres groupes algaux se développent, en particulier les chlorococcales Scenedesmus ecornis Chodat $(9,4 \%)$ et Crucigenia apiculata $(2,6 \%)$, et la diatomée Cyclotella ocellata $(4,8 \%)$, mais ces derniers contribuent faiblement à la biomasse totale en raison de leurs biovolumes relativement faibles. 


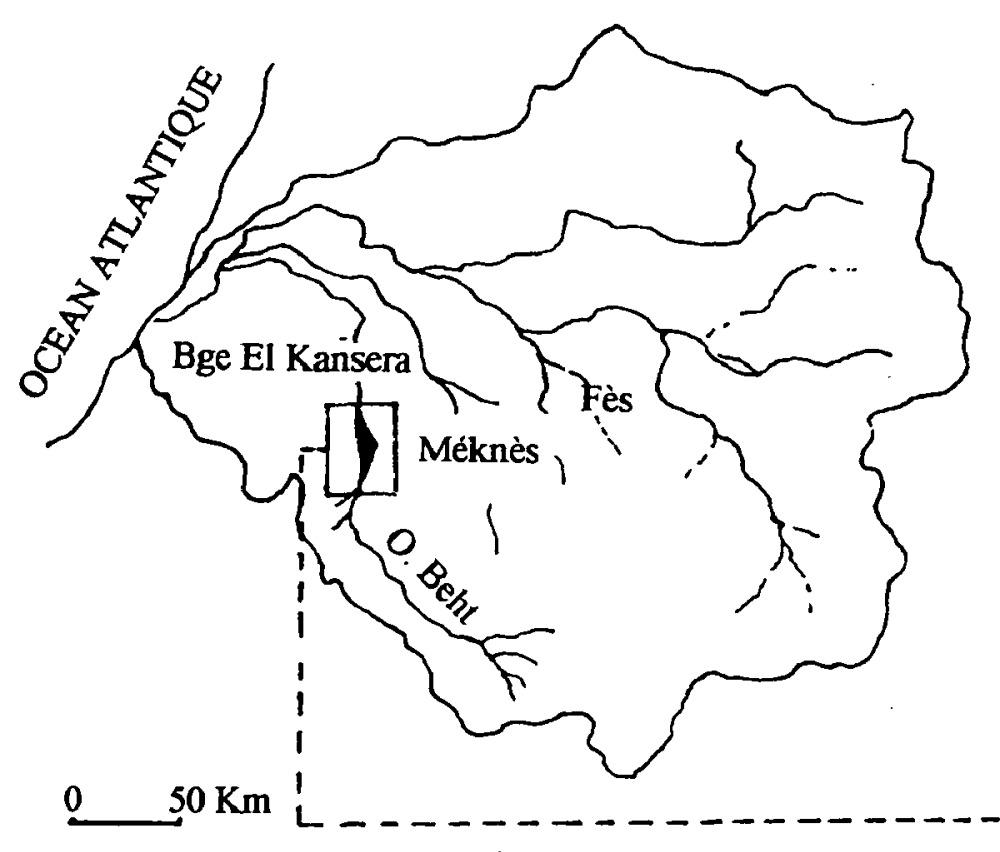

$\uparrow$

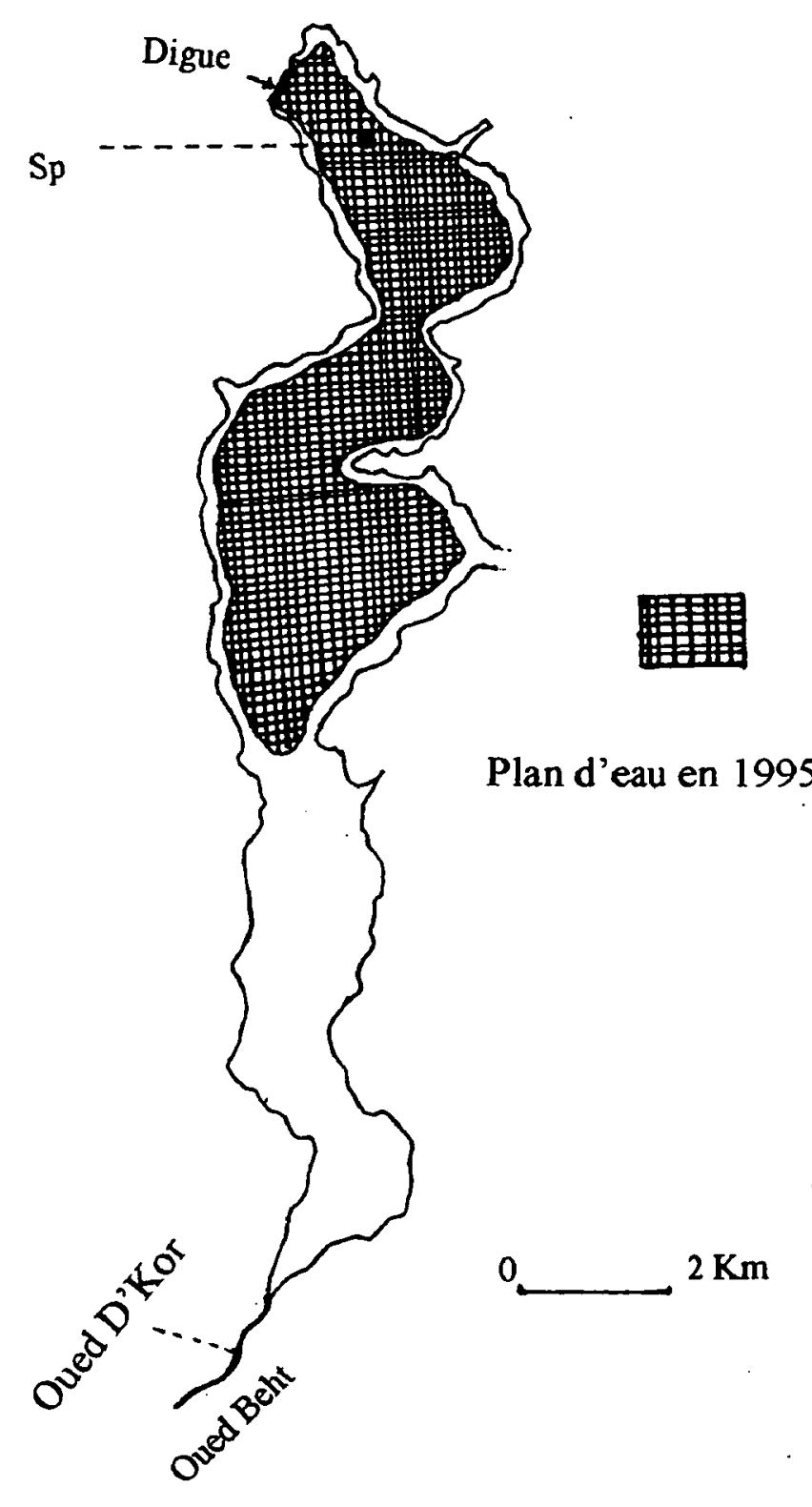

Fig.1. Situation géographique et carte de la retenue de barrage El Kansera. Sp : station de prélèvements.

Fig.1. Geographical situation and map of the lake-reservoir El Kansera. Sp : sampling site.

\subsection{Dynamique saisonnière de quelques espèces do- minantes}

\subsubsection{Microcystis aeruginosa Kütz. (Fig. 8)}

Cette espèce coloniale est autochtone dans la retenue ; Gayral l'avait déjà signalée en 1954. En 1995, elle présente un bloom très important au mois de juin dans la station barrage, où elle se cantonne dans les couches superficielles $(-0,5$ à $-2 \mathrm{~m})$, les plus chaudes $\left(27^{\circ} \mathrm{C}\right)$ et les plus ensoleillées. La transparence de l'eau en cette période est très faible (Fig.2), limitant ainsi la pénétration de la lumière dans la colonne d'eau. Les teneurs en sels nutritifs montrent un épuisement des nitrates sur toute la colonne d'eau (Fig.5A), en même temps qu'un enrichissement du milieu en éléments phosphorés (Fig.5B et $\mathrm{C}$ ). En réalité, la faible profondeur de la colonne d'eau durant cette année sèche (Fig.2), associée aux vents quasi-permanents régnant au niveau de la retenue (Chergui), contribueraient en bonne partie à la resuspension des sédiments fortement enrichis en phosphore, et à l'enrichissement du compartiment aquatique en éléments phosphorés (Piersen \& Weyhenmeyer 1994, Derraz 1995). Parallèlement, et sous . l'action des vents de direction Est (Chergui), les colonies «flottantes» de Microcystis forment des tapis étalés sur plusieurs hectares vers l'amont de la retenue, atteignant des valeurs maximales de $450 \mathrm{mgl}^{-1}$. 


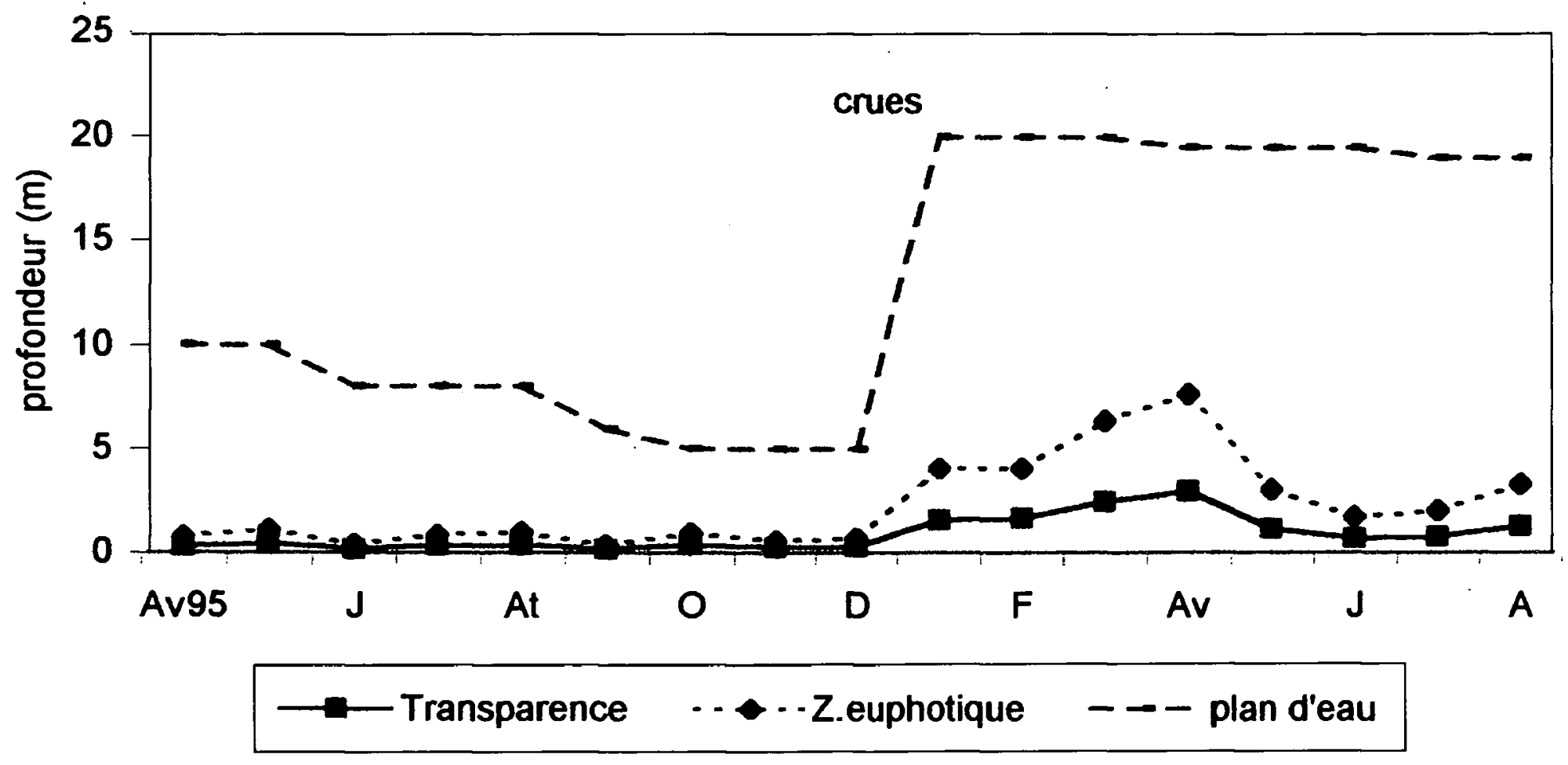

Fig.2. Variations temporelles de la transparence de l'eau, de la zone euphotique et du niveau du plan d'eau de la retenue. Fig.2. Seasonal variations of water transparency, euphotic zone and water level of the lake-reservoir.

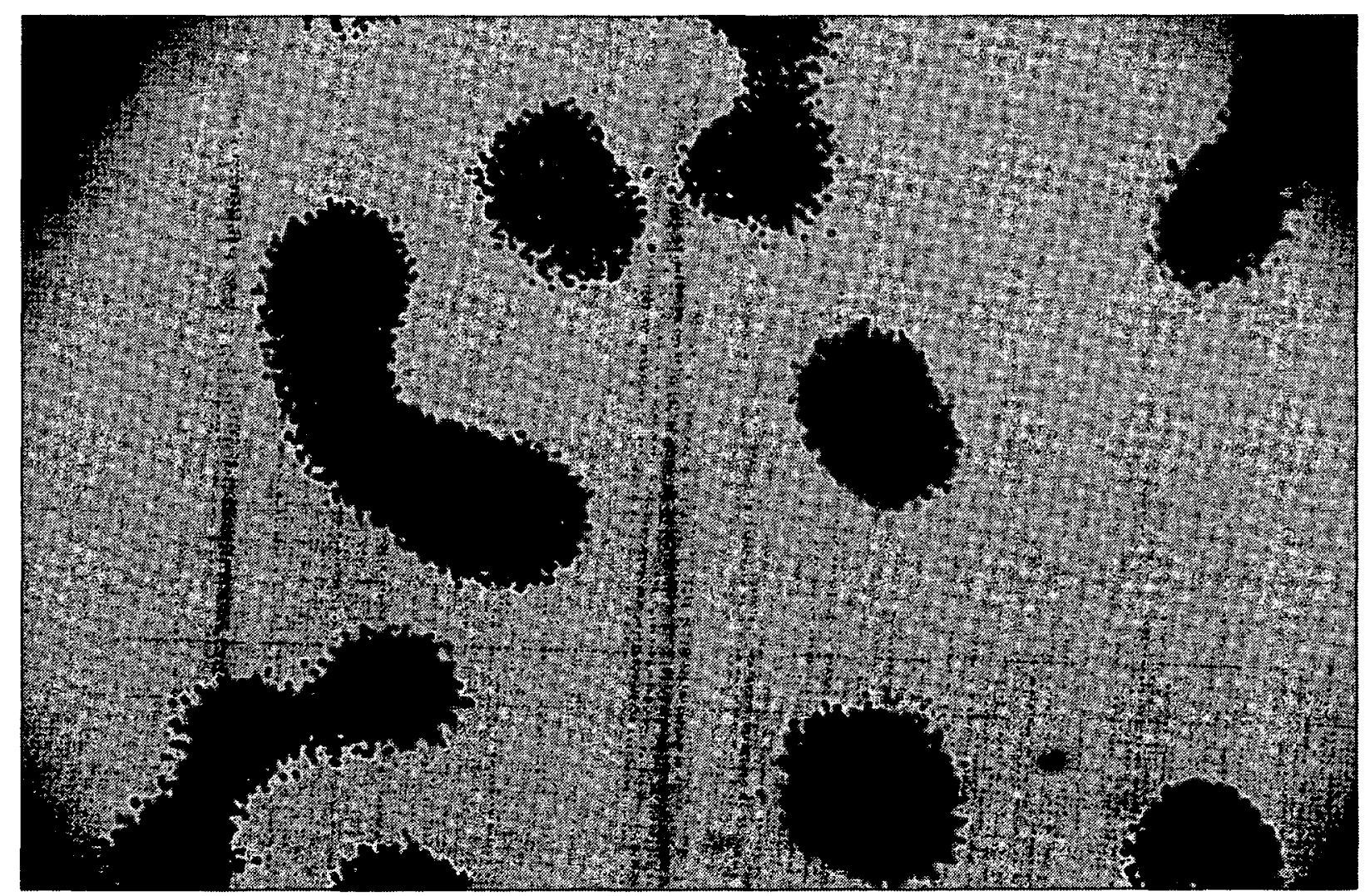

$10 \mu \mathrm{m}$

Fig.3. Colonies de Microcystis aeruginosa (Gr.*100).

Fig.3. Colonies of Microcystis aeruginosa (Gr.*100). 


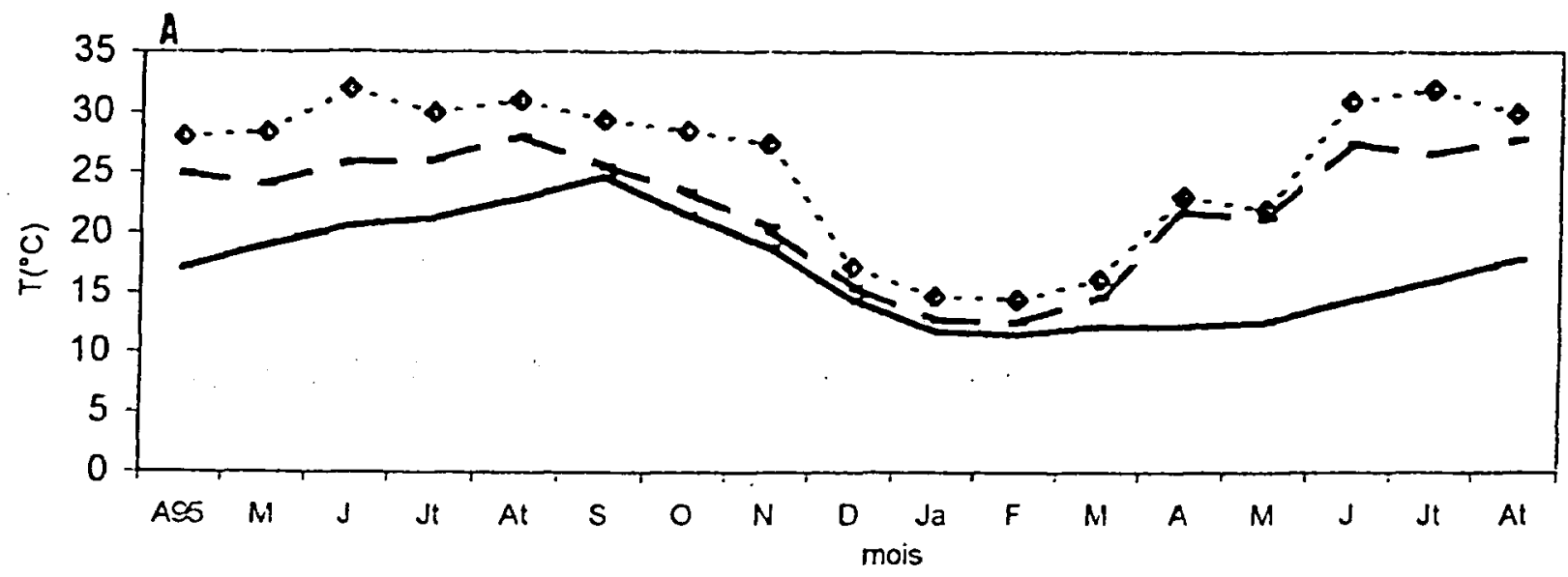

$$
\cdots \bullet-T \text { (air) }-\mathrm{T} \text { (surface) } \longrightarrow \text { (fond) }
$$

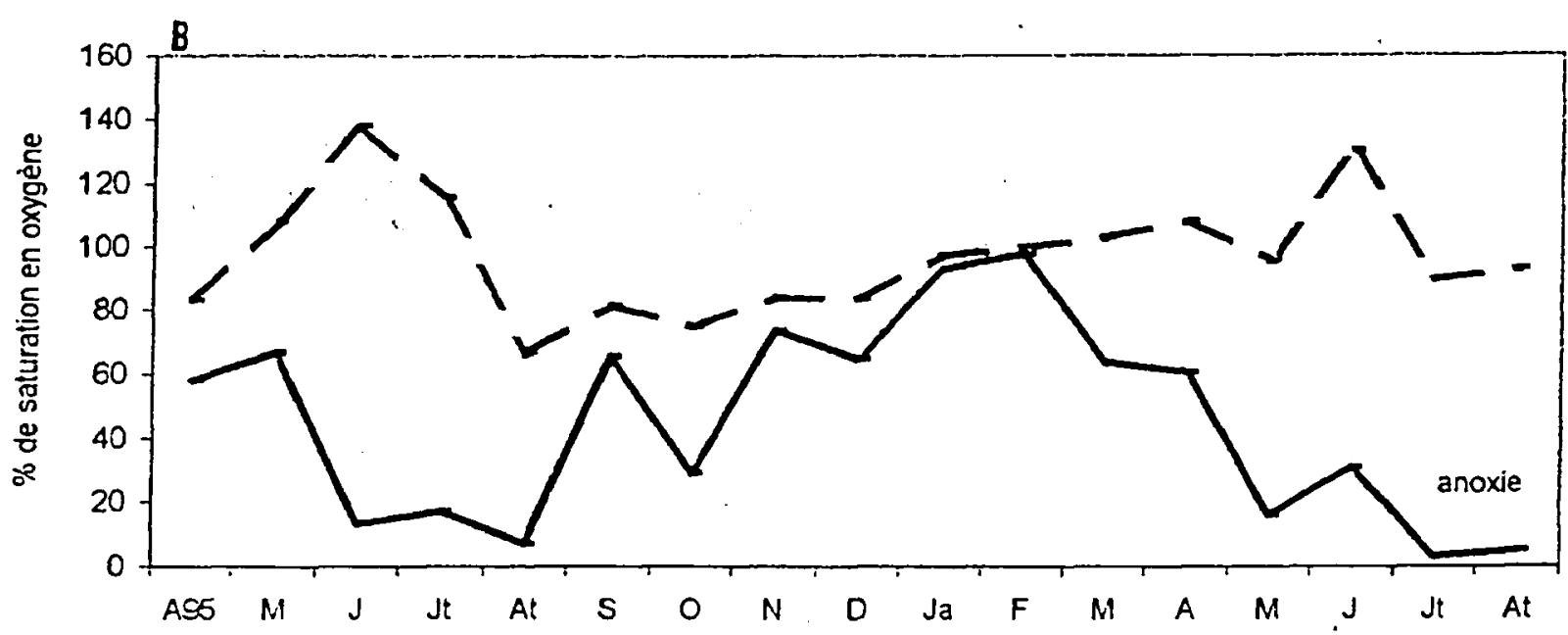

Fig.4. Evolution temporelle de la température et de l'oxygène dissous (\% de saturation) à la surface et au fond de la retenue.

Fig.4. Temporal evolution of temperatur and dissolved oxygen (\% of saturation) at the surface and bottom of the lake-reservoir.

Tableau 1. Principales caractéristiques de la retenue El Kansera.

Table 1. Main features of El Kansera lake-reservoir.

Année de mise en eau

Superficie du bassin versant

Province

Fonction

Type

Hauteur sur fondation

Longueur en crête

Superficie du plan d'eau

Capacité totale

Longueur moyenne

Profondeur maximale

Profondeur moyenne

Volume du barrage

Temps de séjour de l'eau
1935

$4500 \mathrm{~km}^{2}$

Sidi Slimane

Energie, Irrigation, Eau potable

Béton poids et contreforts

$68 \mathrm{~m}$

$170 \mathrm{~m}$

$18 \mathrm{~km}^{2}$

$265,8 \mathrm{Mm}^{3}$

$14 \mathrm{~km}$

$25 \mathrm{~m}$

$16,5 \mathrm{~m}$

$166.000 \mathrm{~m}^{3}(67-265)$

15,7 mois $(13-28,5)$ 

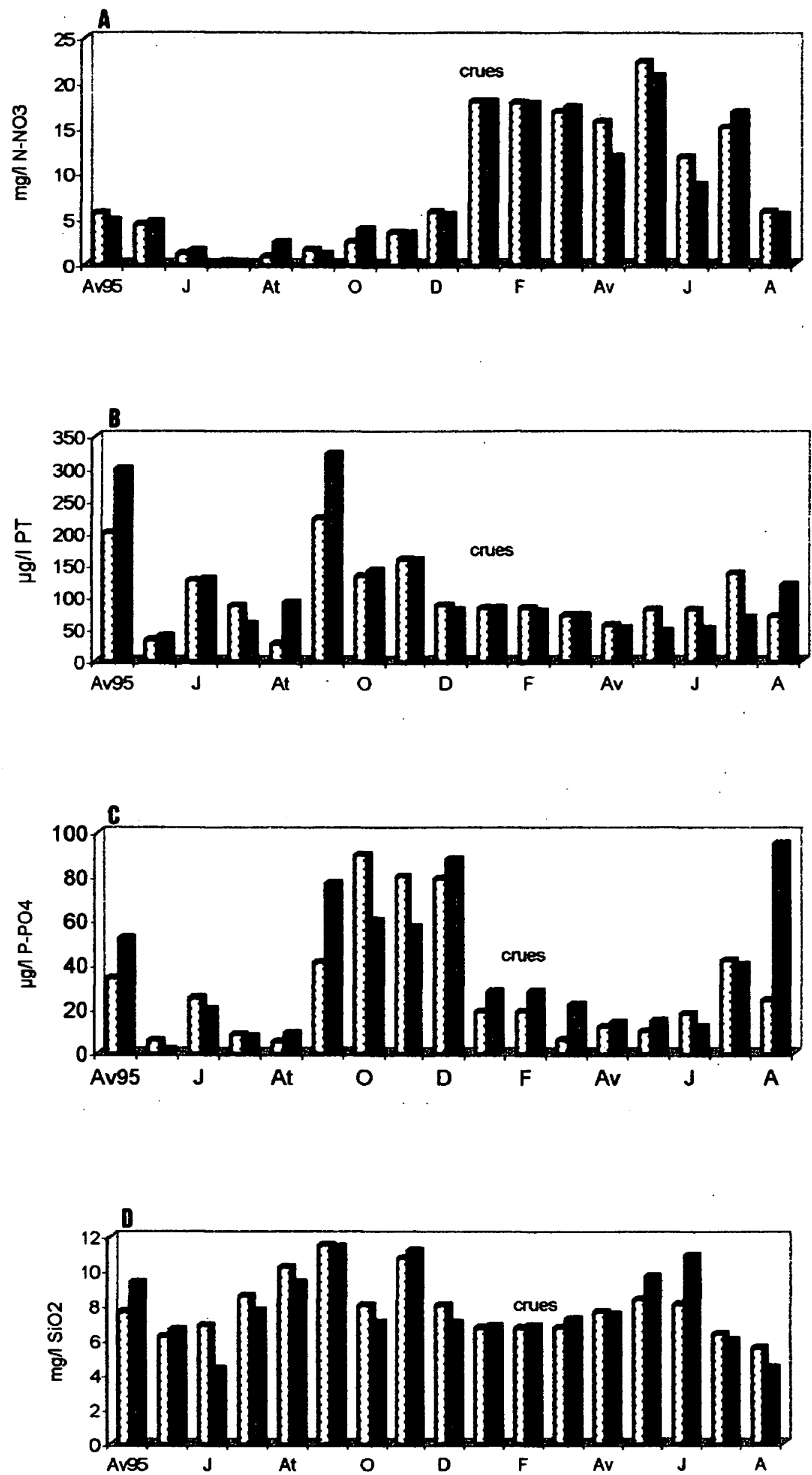

\section{曰surface}

Fig.5. Evolution saisonnière de l'azote nitrique N-NO3 (A), du phosphore total PT (B), des orthophosphates P-PO4 (C), et de la silice SiO2 (D), en surface et au fond de la retenue.

Fig.5. Seasonal variation of nitrate nitrogen N-NO3 (A), total phosphorus (B), phosphorus P-PO4 (C) and silica SiO2 (D) at the surface and bottom of the lake-reservoir. 


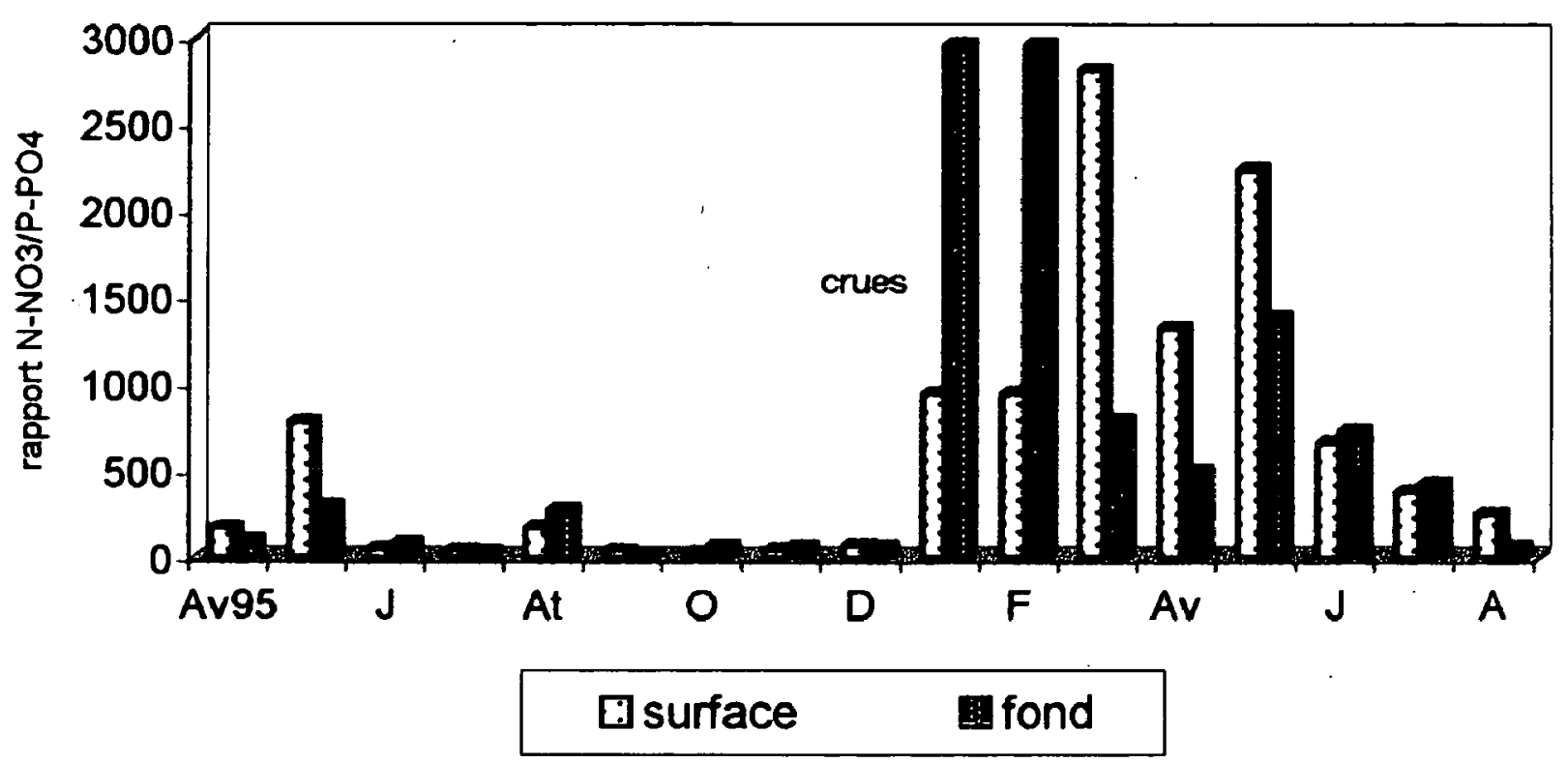

Fig.6. Variations saisonnières du rapport N-NO3/P-PO4.

Fig.6. Seasonal variations of N-NO3/P-PO4.

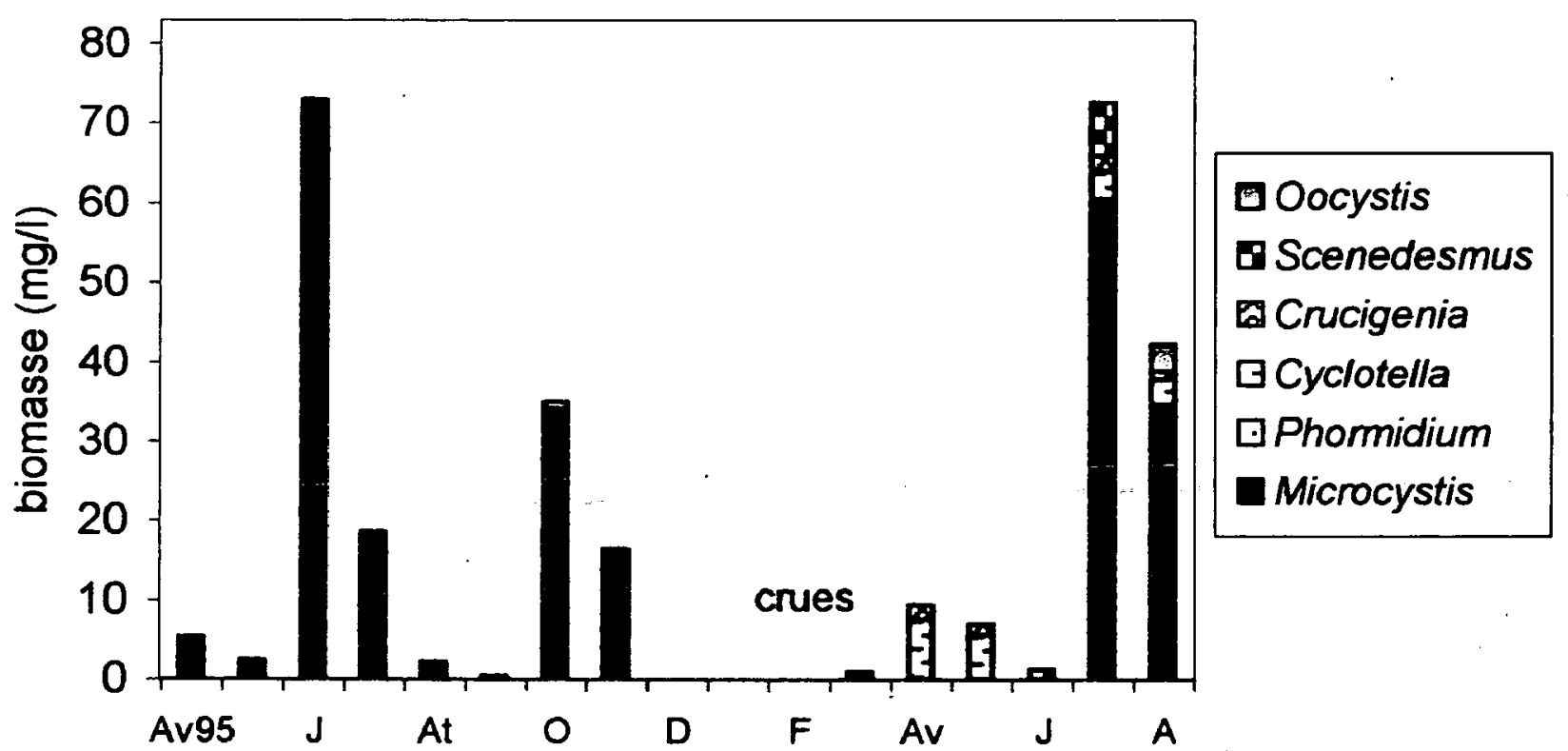

Fig.7. Evolution saisonnière de la biomasse algale (mg/l de poids frais) dans la zone euphotique.

Fig.7. Seasonal development of the algal biomass ( $\mathrm{mg} / \mathrm{l}$ of fresh weight) in the euphotic zone.

Varis (1993) a également observé des blooms à Microcystis dans des lacs eutrophes, turbides et peu profonds (3 à $10 \mathrm{~m}$ ), à des températures élevées, et malgré une forte réduction des nitrates.

En saison automnale (octobre), un nouveau bloom à Microcystis est enregistré. Malgré l'homogénéité thermique qui caractérise les eaux de la retenue en cette période $\left(22^{\circ} \mathrm{C}\right.$ sur toute la colonne d'eau), les colonies se maintiennent en surface, à $-0,5 \mathrm{~m}$ notamment. Cette espèce est en effet dotée d'un pouvoir de flottabilité à la surface de l'eau, grâce à la présence de pseudo-vacuoles (Zohary \& Robart 1989, Konopka et al. 1993). Il semble que la faible transparence de l'eau, qui limi- te la pénétration de la lumière dans la colonne d'eau, soit à l'origine de cette distribution. Au même moment, les teneurs en éléments phosphorés montrent une augmentation sensible (Fig.5B et C), qui serait liée à la remise en suspension des éléments nutritifs durant le brassage automnal. Ces observations sont en accord avec celles de Köhler (1992), Varis (1993) et Derraz (1995), qui ont montré également l'importance de la lumière et du phosphore sur le développement de cette espèce.

L'année suivante, Microcystis recolonise le milieu un peu plus tardivement (juillet), en formant un nouveau bloom dans la station barrage. En cette période, 

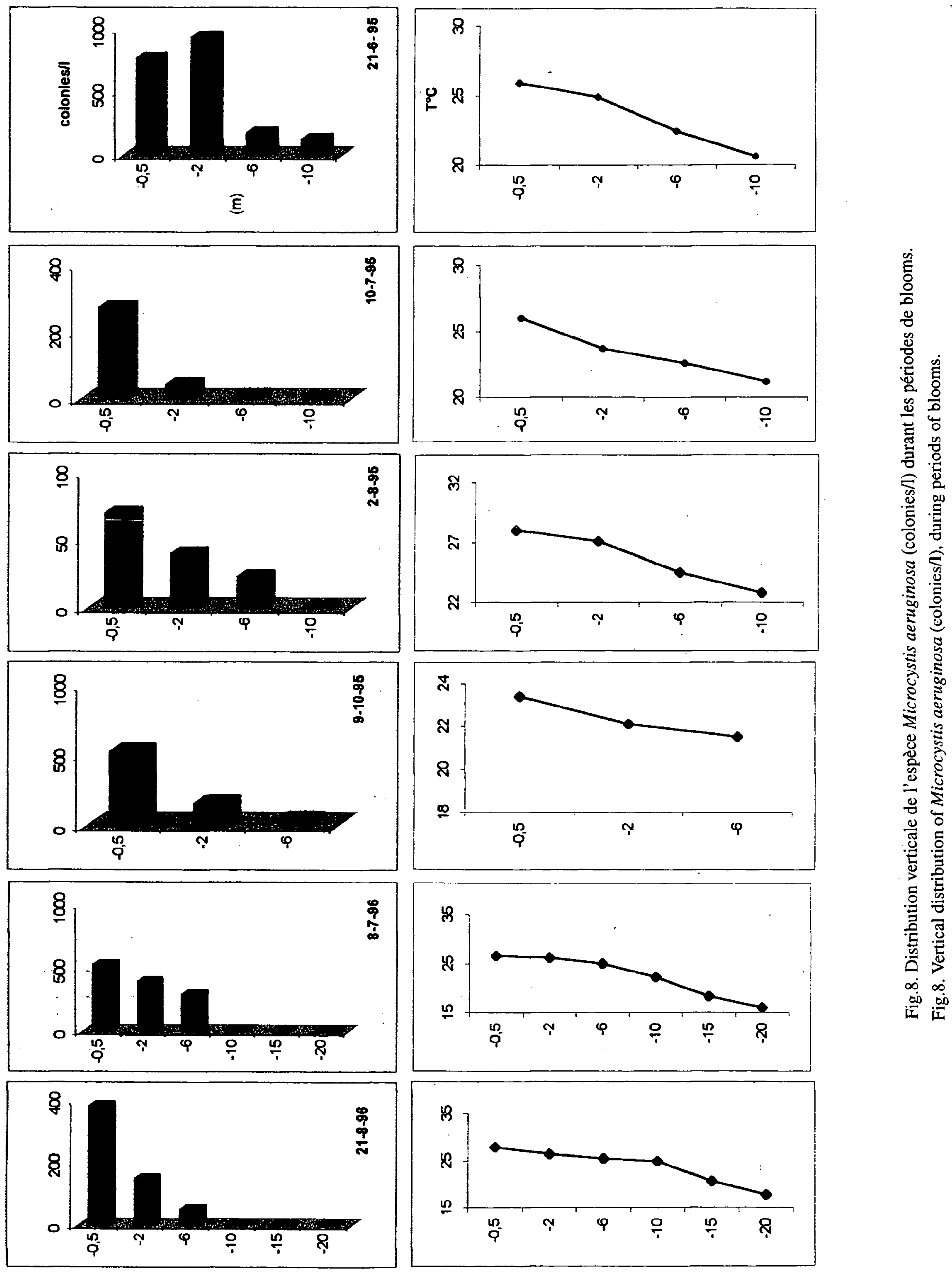
les colonies se concentrent dans les couches épilimniques $(-0,5$ à $-6 \mathrm{~m})$. Cette période coïncide avec une nouvelle phase de stratification thermique, séparant un épilimnion chaud $\left(25 \mathrm{à} 27^{\circ} \mathrm{C}\right)$ d'un hypolimnion relativement froid $\left(16\right.$ à $\left.18^{\circ} \mathrm{C}\right)$. Les teneurs en nutriments montrent des valeurs importantes en nitrates durant toute l'année (Fig.5A), qui seraient liées aux apports allochtones importants (pluies hivernales et crues de l'oued Beht). En même temps, un enrichissement du milieu en éléments phosphorés est observé en saison estivale (Fig.5B et C). Zohary \& Robarts (1989) ont également noté dans le réservoir Hartbeesport, en Afrique du Sud, un développement intense de cette cyanobactérie, en période de haute température et de forte lumière, et en présence de teneurs élevées en nutriments azotés et phosphorés. Cet apport en phosphore, d'origine interne (Derraz 1995), serait lié à une libération par le sédiment anoxique des éléments phosphorés. Ce phénomène a déjà été observé dans d'autres retenues marocaines, telle que la retenue $\mathrm{Al}$ Massira (Dafir 1997).

\subsubsection{Cyclotella ocellata Pant. (Fig. 9)}

Cette diatomée, seconde espèce autochtone dans la retenue (Gayral 1954), présente un cycle de développement différent d'une année à l'autre. En 1995, elle semble très affectée par les conditions de sécheresse (températures élevées durant toute l'année et carence en nitrates notamment). Ainsi, en dépit de fortes concentrations en silice (Fig.5D), elle n'apparaît qu'en saison automnale, mais avec une biomasse négligeable (Fig.7). Cette période coïncide, de plus, avec le développement dans le lac d'une grande biomasse zooplanctonique herbivore. La mauvaise qualité de la nourriture algale en cette période (Cyanobactéries) fait que le broutage est «ciblé» essentiellement sur cette petite diatomée, facilement ingérée par le zooplancton (Moncef 1993).

Au printemps1996, suite à l'enrichissement du milieu en éléments nutritifs azotés (Fig.5A) et à l'augmentation de la température printanière, cette diatomée recolonise la retenue avec des densités très élevées. Elle se développe de façon homogène sur toute la colonne d'eau, avec une légère dominance dans les couches superficielles $(-0,5$ à $-2 \mathrm{~m})$, où les températures varient de 20 à $21,7^{\circ} \mathrm{C}$. Ces observations sont en accord avec les résultats de Capblancq (1972), d'Hermann (1980) et de Loudiki (1990), qui notent que les espèces du genre Cyclotella, apparaissent comme des formes héliophiles, qui se développent de façon optimale lorsque la température avoisine $20^{\circ} \mathrm{C}$.
Au mois de juin, le déclin de Cyclotella coïncide avec un nouveau pic de zooplancton dans le lac. Cet effet de broutage est accentué par l'introduction, dans la retenue, de carpillons argentés de Chine (Hypophthalmichtys molitrix) à régime herbivore (ONEP 1996). Dès le mois de juillet, Cyclotella présente un second pic de développement, entre -6 et $-10 \mathrm{~m}$ de profondeur. Ce pic coïncide avec l'installation de la thermocline à ce niveau ; il apparaît que la diatomée progresse au niveau de la zone euphotique, pour fuir les fortes radiations et les hautes températures estivales $\left(>25^{\circ} \mathrm{C}\right)$. Ce même phénomène a été observé dans le lac de Pareloup (Jrad 1989) et dans la retenue Hassan Ier (Loudiki 1990). Au mois d'août, Cyclotella persiste sur toute la colonne d'eau, mais en réalité, la majorité des cellules observées sont moribondes à cause du phénomène de photooxydation (Cherifi 1992), ou d'intoxication par les toxines de Microcystis (Zhenrong et al. 1993, Enrisken \& Moestryp 1997).

\section{Discussion}

L'étude du phytoplancton de la retenue El Kansera, entre avril 1995 et août 1996, a permis de dégager les quatre points suivants :

1) Le peuplement algal est peu diversifié (59 taxons) et ressemble largement à celui inventorié dans d'autres retenues marocaines. Sur le plan quantitatif, quelques espèces seulement semblent jouer un rôle déterminant, en particulier la cyanobactérie Microcystis aeruginosa.

2) A l'échelle de la période, le suivi du compartiment algal met en évidence de profondes modifications d'une année à l'autre, dans la composition et la structure du peuplement. Ces variations coïncident avec les variations climatiques très prononcées qui ont caractérisé la période d'étude. Ainsi, les cyanobactéries dominent en 1995 (été-automne) et sont représentées essentiellement par l'espèce coloniale Microcystis aeruginosa. Les faibles valeurs du rapport N-NO3/PPO4 montrent que l'azote remplace le phosphore comme facteur limitant (Fig.6). Ces valeurs peuvent expliquer la prédominance des cyanobactéries en cette période. De même, Lewis (1983) explique la formation des blooms à Microcystis par la grande adaptation de cette algue à la limitation des eaux en azote. De plus, cette cyanobactérie paraît la mieux adaptée pour l'assimilation et le stockage du phosphore dans cet écosystème, par comparaison aux autres cyanobactéries Phormidium, Oscillatoria ou Aphanizomenon. L'année suivante, après des apports allochtones importants (pluies' hivernales et crues de l'oued Beht), ce rapport atteint des valeurs beaucoup plus élevées, indiquant que c'est le phosphore qui est le facteur limitant en 

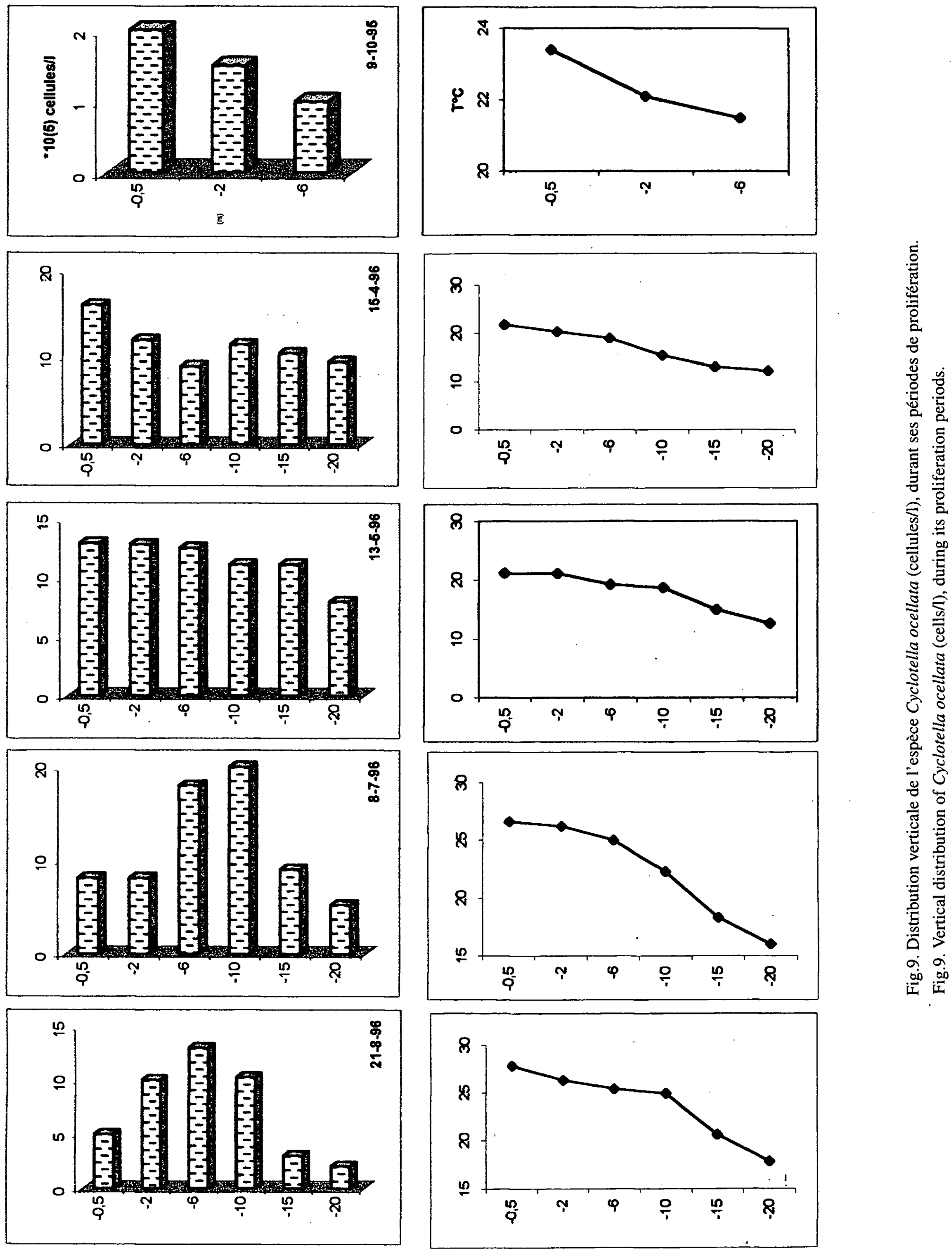
1996. Cela induit une plus grande diversification spécifique algale et la prolifération de nouveaux groupes, en particulier des diatomophycées (Cyclotella ocellata) et des chlorococcales (Crucigenia apiculata, Scenedesmus ecornis, Oocystis lacustris...). Toutefois, en saison estivale, une nouvelle diminution du rapport $\mathrm{N}$ NO3/P-PO4 est à l'origine d'un nouveau bloom à $M i$ crocystis.

3) La répartition verticale des biomasses dans la retenue est déterminée par le régime thermique et indirectement par les changements saisonniers des conditions climatiques (température et lumière). L'accentuation du gradient thermique en saison estivale entraîne une distribution très hétérogène entre l'épilimnion et l'hypolimnion. Ainsi, les maxima de biomasse de $M i$ crocystis se situent dans l'épilimnion, zone la plus chaude et la plus ensoleillée. La mise en place de la stratification thermique semble être un élément fondamental pour le développement de cette cyanobactérie. En revanche, les maxima de Cyclotella plus ou moins homogènes en saison printanière, se concentrent vers $-10 \mathrm{~m}$ en saison estivale, fuyant les températures élevées. Cette espèce semble régie par plusieurs facteurs dans le lac-réservoir, en particulier la température, la disponibilité des nutriments azotés, le broutage (zooplancton et poissons planctonophages), ainsi que la compétition avec Microcystis aeruginosa. A l'inverse, cette cyanobactérie est très compétitive et prolifère dans le lac-réservoir au détriment des autres espèces, dès que les conditions du milieu deviennent difficiles (températures élevées, carence en nutriments...). $M i$ crocystis aeruginosa est de plus, rarement broutée du fait de son caractère toxique reconnu; l'observation de plusieurs cadavres de poissons planctonophages en été 1996, après leur introduction dans le lac, le confirme (ONEP 1996).

4) Durant la période d'étude, l'état trophique de la retenue, déterminé à partir des paramètres de biomasse de poids frais (Vollenweider 1969), semble évoluer d'une année à l'autre : hypereutrophe en 1995, la retenue EL Kansera devient, par la suite, méso-eutrophe, avec une tendance nette à l'eutrophie en saison estivale. De même, les paramètres de classification du modèle de l'O.C.D.E (1982), en particulier le phosphore total, la transparence de l'eau et la chlorophylle, montrent une évolution similaire.

\section{Conclusion}

Les fortes variations climatiques enregistrées entre 1995 et 1996 (transition d'une année de sécheresse avec une année pluvieuse) seraient à l'origine d'une amélioration relative de la qualité des eaux de la rete- nue El Kansera. Cependant, le caractère eutrophe de ce lac-réservoir semble relativement stable et "persistant», avec notamment la réapparition des blooms à Microcystis en été 1996. Ces considérations montrent la nécessité d'intervention pour la sauvegarde de la qualité des eaux de cette retenue, qui sont destinées entre autres, à l'alimentation en eau potable de deux villes du pays Khemisset et Tiflet. Cela représente une «menace» permanente pour le consommateur, si l'on tient compte des effets hépathotoxiques voire même neurotoxiques de cette algue (Thébault et al. 1995). Ainsi, si l'on veut éviter des «dégâts» humains, comme cela a été observé au centre d'hémodialyse du Brésil en 1996 suite à une intoxication par Microcystis aeruginosa, des moyens de lutte contre la prolifération de cette cyanobactérie en particulier, devraient être appliqués, selon le protocole suivant :

- un contrôle des apports en nutriments phosphorés en amont de la retenue, et plus particulièrement dans l'oued D'Kor, affluent de l'oued Beht (Fig.1), qui véhicule les rejets domestiques de Khemisset et qui est très eutrophisant au point de vue phosphore (Derraz 1995);

- une introduction de $\mathrm{CO}_{2}$ libre au fond de la retenue, ce qui favoriserait le développement d'autres groupes phytoplanctoniques au détriment de cette cyanobactérie, fixatrice de $\mathrm{CO}_{2}$ atmosphérique ;

- une déstratification artificielle au printemps, qui permettrait de retarder la stratification et de réduire la réponse trophique estivale des cyanobactéries notamment ;

- une réduction du temps de séjour des eaux dans la retenue, qui permettrait de maintenir la réponse trophique à un niveau relativement bas, de par les importantes turbulences hydrodynamiques crées dans le milieu.

\section{Remerciements}

Ce travail a été réalisé dans le cadre d'une action intégrée entre la faculté des Sciences Aïn Chock de Casablanca (Laboratoire Biologie et Santé), et le laboratoire Public d'Essais et d'Etudes (Centre de recherche et d'études sur la pollution de l'eau et de l'air, CEREP). Nous tenons à remercier personnellement le Docteur Jabry, directeur du CEREP, qui nous a accueilli dans ce laboratoire, le Professeur Fahde de la Faculté des Sciences I de Casablanca pour son appui et ses conseils, ainsi que le Professeur Loudiki de la Faculté des Sciences de Marrakech qui nous a aidé à confirmer les espèces algales.

\section{Travaux cités}

Abouzaid H. \& Foutlane A. 1986. - L'eutrophisation de quelques lacs-réservoirs au Maroc. Revue marocaine du Génie Civil et des Travaux Publics, 9 (83) : 4-58.

Afnor 1994. - Recueil des normes françaises, eaux-méthodes d'essais : $350 \mathrm{p}$ 
Balvay G., Gawler M \& Pelletier J.P. 1990. - Lake trophic status and the development of the clear-water phase in Lake Geneva. In Large lakes - ecological structure and functiun : $691 \mathrm{p}$.

Bouterfas R. 1990. - Contribution à la connaissance du fonctionnement d'un lac-réservoir récemment mis en eau : la retenue Hassan I $^{\text {er }}$ (Maroc). Relations avec le bassin versant : hydrochimie et phytoplancton. Thèse $3^{\text {ème }}$ cycle, Faculté Semlalia, Univ. Cadi Ayyad, Marrakech : 353 p.

Capblancq J. 1972. - Phytoplancton et productivité primaire de quelques lacs d'altitude dans les pyrénées. Annls.Limnol., 8 : 231-321.

Cherifi O. 1992. - Evolution et dynamique du phytoplancton en relation avec certains paramètres biotiques et abiotiques au niveau de la retenue Lalla Takerkoust (Maroc). Thèse $3^{\text {ème }}$ cycle. Univ.Cadi Ayyad, Marrakech : 158 p.

Dafir J.E. 1997. — Organisation et fonctionnement des écosystèmes aquatiques : barrage Al Massira, Baie de Dakhla, Lagune de Nador (gestion et préservation). Application de la dynamique du phosphore (flux de fixation/relargage à l'interface eau-sédiment, spéciation des différentes fractions mobilisables). Thèse d'Etat, Faculté des Sciences, Université Hassan II, Aïn Chock, Casablanca : $635 \mathrm{p}$.

Dauta A. 1982. - Conditions de développement du phytoplancton. Etude comparative du comportement de huit espèces en culture. IDétermination des paramètres de croissance en fonction de la lumière et de la température: Annls Limnol., 18 (3) : 263-292.

Derraz M. 1995. - Etude de l'eutrophisation de la retenue de barrage El Kansera (Maroc). Caractéristiques physico-chimiques, biodisponibilité du phosphate sédimentaire, écophysiologie de $M i$ crocystis et relation avec les paramètres environnementaux. Thèse d'Etat. Univ. Moulay Ismaîl. Méknès : $125 \mathrm{p}$.

Devaux J., Lair N. \& Amblard C. 1983. - Un écosystème lacustre profond : le lac Pavin. In Problèmes d'écologie : structure et fonctionnement des systèmes limniques, Lamotte M. \& Bourlière F., (eds) Masson, Paris : 1-49.

Enrisken H. \& Moestryp O. 1997. - Seasonnal variations in microcystin contents of Danish cyanobacteria. Nat.Toxins, $97 ; 5$ (3) : 99-106.

Essini A. 1987. - Zone d'épandage des eaux usées de la ville de Marrakech (Maroc) ; biotopes aquatiques, peuplement algal et qualité des eaux. Thèse $3^{\text {ème }}$ cycle, Univ. Cadi Ayyad, Marrakech : $172 \mathrm{p}$.

Gala W.R. \& Giesy J.P. 1991. - Effects of U.v radiations on primary production of natural phytoplankton assemblage in Lake Michigan. Ecotoxicol. Environ. Saf., $91 ; 22$ (3) : 345-611.

Gayral-Engerbaud P. 1954. - Recherches phytolimnologiques au Maroc. Thèse de Doctorat, Univ. Paris : 156p.

Hasnaoui M. 1992. - Biomasse, production primaire et distribution horizontale et verticale du phytoplancton dans le lac-réservoir Hassan Ier (Maroc). Thèse $3^{\mathrm{ème}}$ cycle. Univ. Cadi Ayyad. Marrakech : 104 p.

Hermann H. 1980. - Interesting planctonic forme littoral algae from waters in the district haule G.D.R. 3, Arch. Protistenk, 123 : 349-357.

Jrad A. 1989. - Phytoplancton et production primaire dans le lac de Pareloup (Aveyron). Thèse $3^{\text {ème }}$ cycle, Univ. P. Sabatier, Toulouse : $166 \mathrm{p}$.

Köhler J. 1992. — Influence of turbulent mixing on growth and primary production of Microcystis aeruginosa in the hypertrophic bautzen reservoir. Arch.Hydrobiol., 123 (4) : 413-429.

Konopka A.E., Klemer A.R., Walsby A.E. \& Ibelings B.W. 1993. Effects of macronutrients upon buoyancy regulation by metalimnetic Oscillatoria agardhii in Deming Lake, Minnesota. J. Plankton. Res., 15 (9) : 1019-1034.
Lahlou A. 1991. - Etude actualisée de l'envasement des barrages au Maroc. Rev. Sci. Eau, 6 (3) : 337-356.

Lair N. \& Sargos 1981. - Dynamique des populations planctoniques en Loire moyenne. Annls Stat. biologique de Besse en Chandesse, $15: 60-114$

Lamotte M. \& Bourlière F. 1971. — Problèmes d'écologie : l'échantillonnage des peuplements animaux des milieux aquatiques. Ed. Masson et Cie, Paris : 294 p.

Lampert W., Fleckner W., Rai H., Taylor B. 1986. - Phytoplankton control by grazing zooplankton : a study on the spring clear-water phase. Limnol.Oceanogr. 31 (3) : 478-490.

Le Cohu R., Comoy N., Guitard J. \& Brabet J. 1991. —Périodicité du phytoplancton dans un réservoir de moyenne profondeur ; le lac de Pareloup (Massif Central) France. Annls Limnol., 27 : 197-214.

Lewis W.M. 1983. - Interception of atmospheric fixed nitrogen as an adaptative advantage of scum formation in blue-green algae. $\mathrm{J}$. phycol. $19: 534-536$

Lohmann H. 1908. - Untersuchungen zur Festellung des vollstandigen Gehaltes des Meeres an Plankton. Wiss Meeresunters, Abt. Kiel N. F., $10: 132-170$.

Loudiki M. 1990. — Etude limnologique d'un hydrosystème récemment aménagé dans la région de Marrakech dans la région de Marrakech (Maroc). Apports du bassin versant, hydrochimie, algues et macrophytes. Thèse Doctorat, Univ. d'Aix-Marseille : $335 \mathrm{p}$.

Maïffi M. 1988. - Impact des rejets des eaux usées de la ville de Marrakech sur l'oued Tensift ; les Diatomées, indicateurs biologiques. Thèse de Doctorat, Univ. Paris : $173 \mathrm{p}$.

Malki M. 1994. - Etude des communautés phytoplanctoniques et caractéristiques physico-chimiques des eaux du lac-réservoir $\mathrm{Al}$ Massira. Thèse de Doctorat, Univ. Hassan II, Casablanca : 168 p.

Moncef M. 1993. - Etude du peuplement de Copépodes et de larves de Chaoborus de la retenue AL Massira (Maroc). Thèse Doctorat, Univ. Caddi Ayyad, Marrakech : 315 p.

Mouhri K. 1987. - Répartition et association des Desmidiées (Chlorophycées, Zygnématales) dans différentes zones géographiques de la région de Marrakech (Maroc). Thèse $3^{\text {ème }}$ cycle, Univ. Caddi Ayyad, Marrakech : $152 \mathrm{p}$.

ONEP 1996. - Rapport de l'Office National de l'Eau Potable sur les retenues de barrage marocaines : $30 \mathrm{p}$.

Piersen D.C. \& Weyhenmeyer G.A. 1994. - High resolution measurements of sediment resuspension above an accumulation bottom in a stratified lake. Hydrobiologia, $284: 43-57$.

Quiblier-Lloberas C., Amblard C. \& Pepin D. 1996. - Impact of grazing on phytoplankton in lake Pavin (France) : contribution of different zooplankton groups. J. Plankton Res., : 305-322.

Reynolds C.S. 1984. - Phytoplankton periodicity : the interactions of form, function and environmental variability. Freshwater Biol., $14(2): 111-142$.

Thébault L., Lesne J. \& Boutin J.P. 1995. — Les Cyanobactéries, leurs toxines et les risques pour la santé. Medecine Trop., 55 : 375-380.

Utermöhl H. 1958. - Zur Vervollkommung der quantitativen Phytoplanton Methodik. Mitt. Int. Verein. Theor. Angew. Limnol.,9 : 1-38.

Varis O. 1993. - Cyanobacteria dynamics in a restored Finnish lake : a long term simulation study, Hydrobiologia, $268: 129-145$.

Vollenweider R.A. 1969. - A manual of methods for measuring primary production in aquatic environment. I.B.P. Handbook 12, Blackwell Scientific Publication, Oxford : 244 p.

Zhenrong H., Minjuan Y. \& Jiawan H. 1993. - Toxins from Microcystis aeruginosa strain M.8641. Biochem.J., 9 (2) : 168-172.

Zohary T. \& Robart R.D. 1989. - Diurnal mixed layers and longterm dominance of Microcystis aeruginosa, J.Plankton.Res., 11 (1) : 25-48. 